\title{
Hematological Parameters Outperform Plasma Markers in Predicting Long-Term Mortality after Coronary Angiography
}

Crystel M. Gijsberts, MD, PhD ${ }^{1,2}$, Hester M. den Ruijter, PhD¹, Dominique P.V. de Kleijn, PhD ${ }^{1,2,3,4}$, Albert Huisman, $\mathrm{PhD}^{5}$, Maarten ten Berg, $\mathrm{PhD}^{5}$, Mark de Groot, $\mathrm{PhD}^{5}$, Richard H.A. van Wijk, $\mathrm{PhD}^{5}$, Folkert W. Asselbergs, MD, $\mathrm{PhD}^{6,7,8}$, Michiel Voskuil, MD, $\mathrm{PhD}^{6}$, Gerard Pasterkamp, MD, $\mathrm{PhD}^{1,5}$, Wouter W. van Solinge, $\mathrm{PhD}^{5}$, Imo E. Hoefer, MD, $\mathrm{PhD}^{1,5}$

\begin{abstract}
1. Experimental Cardiology Laboratory, University Medical Center Utrecht, Heidelberglaan 100, 3584CX, Utrecht, The Netherlands
2. ICIN-Netherlands Heart Institute, Moreelsepark 1, 3511EP, Utrecht, the Netherlands.

3. Department of Surgery, Yong Loo Lin School of Medicine, National University of Singapore, IE Kent Ridge Road Singapore 119228, Singapore.

4. Cardiovascular Research Institute (CVRI), National University Heart Centre (NUHCS), National University Health System, IE Kent Ridge Road Singapore 119228, Singapore.

5. Department of Clinical Chemistry and Hematology, University Medical Center Utrecht, Heidelberglaan 100, 3584CX, Utrecht, The Netherlands.

6. Cardiology, University Medical Center Utrecht, Heidelberglaan 100, 3584CX, Utrecht, the Netherlands.

7. Durrer Center for Cardiogenetic Research, ICIN-Netherlands Heart Institute, Moreelsepark 1,3511EP, Utrecht, The Netherlands. 8. Institute of Cardiovascular Science, faculty of Population Health Sciences, University College London, Gower St, London WC1E 6BT, United Kingdom.
\end{abstract}

Corresponding author: Imo E. Hoefer, University Medical Centre Utrecht Heidelberglaan 100, 3584CX Utrecht, The Netherlands

Telephone: +318875567490

Fax: +31 8875550900

i.hoefer@umcutrecht.nl

Acknowledgements: We sincerely thank Miss Jonne Hos for her excellent contribution to the UCORBIO cohort.

There are no relationships with the industry to declare. 


\section{Abstract}

High-sensitivity troponin I (hsTnl) and N-terminal pro-brain natriuretic peptide (NTpro-BNP) are predictors of coronary artery disease. Recently, routine hematological parameters emerged as mortality predictors. We examined the predictive value of hematological parameters (from the Utrecht Patient Oriented Database; UPOD) and hsTnl and NTpro-BNP for mortality in a coronary angiography population (Utrecht Coronary Biobank $n=1,913$ ). Using Cox regression, receiver operating characteristics, integrated discrimination improvement (IDI) and continuous net reclassification improvement (cNRI) analysis, we compared the predictive properties of hematological parameters with hsTnl and NTpro-BNP for mortality. During a median follow-up duration of 1.8 years, 77 deaths occurred. A panel of 7 hematological parameters (leukocyte count; reticulocyte mean corpuscular hemoglobin concentration; red blood cell (RBC) green (FL1) fluorescence; \%neutrophils; \%large (>120fL) RBCs, \%monocytes and coefficient of variation of neutrophil complexity) was highly predictive. Added to clinical characteristics, hematological parameters (area under the curve $[A U C]: 0.856, p<0.001, I D I: 0.07, p<0.001, c N R I: 0.37, p<0.001)$ were better predictors than hsTnl (AUC:0.818) or NTpro-BNP (AUC:0.834) alone or combined (AUC:0.834). Hematological parameters may provide mortality risk information following coronary angiography and may be superior to hsTnl and/or NTpro-BNP.

Keywords: coronary artery disease, biomarkers, mortality. 


\section{Introduction}

High-sensitivity troponin $\mathrm{I}^{1}$ (hsTnl) and NT pro-B-natriuretic peptide ${ }^{2}$ (NTpro-BNP) constitute the current clinical gold-standard biomarkers for diagnosis and prognosis in acute myocardial infarction, ${ }^{3}$ stable heart failure, ${ }^{4}$ elective coronary angiography, ${ }^{5}$ stable and unstable coronary artery disease $(C A D)^{6,7}$ and percutaneous coronary intervention ${ }^{8}(\mathrm{PCl})$ patients.

Recently, a different and easily accessible type of biomarker has emerged. Blood cell characteristics (counts and percentages), e.g. from leukocytes 9,10 and red blood cells (RBCs), ${ }^{11}$ harbor prognostic information in diverse patient populations. Such hematological parameters are widely available and measured on a routine basis. Modern automated hematology analyzers automatically perform a whole blood cell count irrespective of the clinical request. While not routinely reported to the physician, the unrequested parameters can be extracted and stored for future reference. In the University Medical Center in Utrecht, blood cell differentiation data from the Abbott Sapphire ${ }^{12}$ hematology analyzer have been stored in the Utrecht Patient Oriented Database (UPOD) ${ }^{13}$ for research purposes. The measured parameters consist of cell counts, cell sizes and other cell properties such as cell complexity and granularity.

As precise estimation mortality risk in CAD patients is key for a patient-specific treatment policy and for accurate patient information, we compared the predictive power of routine hematological parameters with the current clinical standard, hsTnl and NTpro-BNP, for prediction of mortality during two years of follow-up in CAD patients undergoing coronary angiography.

\section{Methods}

\section{Study population}

In this study we analyzed data from the Utrecht Coronary Biobank (UCORBIO) cohort (registered as an observational study at clinicaltrials.gov, identifier: NCT02304744), an observational cohort study of coronary angiography patients in the University Medical Center in Utrecht, the Netherlands. From October 2011 to December 2014, a total of 2,591 patients were enrolled. For the current study, we selected adult ( $>18$ years) patients presenting with 
myocardial infarction (either ST-Segment Elevation Myocardial Infarction or Non-ST-Segment Elevation Myocardial Infarction), chest pain without release of cardiac enzymes (stable or unstable angina), dyspnea on exertion, silent ischemia or screening for non-cardiac surgery with complete case information $(n=1,913)$. Patients with other indications for coronary angiography (coronary anomalies, screening for cardiac surgery or follow-up after heart transplantation) were excluded.

Ethics, consent and permissions

All patients provided written informed consent and the study conforms to the Declaration of Helsinki. The institutional review board of the University Medical Centre Utrecht approved of this study (registration number 11-183).

Data collection

Data collection in the UCORBIO cohort has been described before. ${ }^{14}$ In summary, standardized electronic case report forms were completed at baseline containing age, sex, cardiovascular risk factors, indication for angiography, medication use, angiographic findings and eventual treatment of CAD. The angiographic findings were categorized into 4 groups by the treating interventional cardiologist: no CAD, minor CAD (wall irregularities, $<50 \%$ stenosis), single vessel disease (one vessel with $>50 \%$ stenosis $^{15}$ ) and multi-vessel disease ( 2 or 3 vessels with $>50 \%$ stenosis).

\section{Plasma Biomarkers}

Plasma biomarkers (hsTnl and NTpro-BNP) were measured in thawed EDTA plasma, which had been drawn directly prior to coronary angiography from the arterial sheath, before heparinization and immediately stored at $-80^{\circ} \mathrm{C}$. hsTnl was measured using the STATHigh Sensitive Troponin-I assay on the ARCHITECT i2000 analyzer (Abbott Park, Illinois, USA). NTpro-BNP was measured using a semi-automated ELISA robot (Freedom EVO, Tecan, Switzerland, antibodies: 15C4 and biotinylated 13G12, Hi-test Finland).

Hematological parameters

Hematological parameters were obtained through complete blood count analysis at inclusion from the same blood sample as the plasma biomarkers. Fifty-six routinely measured RBC, leukocyte and platelet parameters from the UPOD database were initially taken into consideration in this study. ${ }^{13}$ All hematological parameters were measured using the Cell-Dyn 
Sapphire ${ }^{16}$ hematology analyzer (Abbott Diagnostics, Santa Clara, CA, USA). This analyzer is equipped with an integrated 488-nm blue diode laser and uses spectrophotometry, electrical impedance, laser light scattering (multi-angle polarized scatter separation), and 3-color fluorescent technology to measure morphological parameters of leukocytes, RBCs and platelets for classification and enumeration. The morphological parameters entail the following 5 optical scatter signals for leukocytes: cell size $\left(0^{\circ}\right.$ scatter, axial light loss $)$, cell complexity and granularity $\left(7^{\circ}\right.$ scatter, intermediate angle scatter (IAS)), nuclear lobularity $\left(90^{\circ}\right.$ scatter, polarized side scatter (PSS)), depolarization $\left(90^{\circ}\right.$ depolarized side scatter (DSS)) and viability (red fluorescence (FL-3), $630 \pm 30 \mathrm{~nm}$ ). Platelets are analyzed using two optical scatter signals: IAS scatter $\left(7^{\circ}\right.$, cell size) and PSS scatter $\left(90^{\circ}\right.$, granularity; internal structure). RBC parameters are measured or calculated based on impedance measurements. Reticulocytes are optically measured using IAS scatter $\left(7^{\circ}\right.$, cell size) and FL-1 fluorescence (RNA content). Throughout this paper, all hematological parameters are reported as multitudes of their standard deviation (SD) to facilitate comparison of effect sizes between parameters as their absolute values vary strongly in their order of magnitude.

\section{Statistical Analysis}

Baseline characteristics are reported as means and standard deviations for continuous variables and percentages for categorical variables, both, for the entire cohort and for survivors and non-survivors separately.

First, we constructed a clinical risk prediction model. Covariates for this model were selected using a backward stepwise Cox regression model for all-cause mortality, which and comprised: age, sex, diabetes, hypercholesterolemia, smoking status, indication for angiography, angiographic $\mathrm{CAD}$ severity, history of $\mathrm{PCl}$, history of acute coronary syndrome (ACS), kidney failure and treatment following angiography. The model with the lowest Akaike information criterion was selected. Assumptions for Cox regression were checked and satisfied.

To determine a predictive panel of hematological parameters we first evaluated mutual correlation of the parameters (total $n=56$ ) by means of hierarchically clustered heatmap analysis, grouping closely related parameters in a cluster. From each cluster of collinear parameters (spearman's $\mathrm{R}>0.6$ or $<-0.6$ ) the parameter with the strongest relation 
with all-cause mortality was selected for further analysis. The remaining parameters $(n=34$, supplemental table 1) were entered in a backward stepwise Cox regression model. Subsequently, the top 10 significant parameters were added to the clinical model (which was coerced to stay in the model) and backward stepwise Cox regression was performed again to determine the final panel of hematological parameters.

Receiver operating characteristics (ROC) analysis was performed to assess the prognostic value of hsTnl, NTpro-BNP and the panel of hematological parameters in addition to the clinical model. The clinical model was entered as a linear predictor to stabilize its predictive value. Next, we evaluated the prognostic value of adding hsTnl, NTpro-BNP or both to the panel of hematological parameters (all on top of the clinical model).

For visualization of the predictive value of the panel of hematological parameters, a linear predictor was constructed and the predicted risk based on this linear predictor was divided into quartiles. Survival, adjusted for the clinical parameters was subsequently plotted for these quartiles.

Internal validation of the predictive properties of the hematological parameters was performed by means of post-estimation parameterwise shrinkage ${ }^{17}$ using the jackknife method (repeating the analysis leaving out 1 observation at a time). For this purpose we used the "shrink" package ${ }^{18}$ for R. Also, optimism-adjusted AUCs were calculated using a bootstrapping method.

Furthermore, continuous net reclassification improvement (cNRI) and integrated discrimination improvement (IDI) measures for the abovementioned comparisons were calculated using the "survIDINRI" package ${ }^{19}$ to assess risk prediction improvement. The cNRI corresponds to the percentage of patients that is correctly reclassified by the addition of a new parameter to the previous Cox model and is calculated by adding the percentage of deceased patients who appropriately had higher predicted risk in the new model to the percentage of alive patients who appropriately had a lower predicted risk in the new model. ${ }^{20}$ Continuous NRI was deemed preferable over categorical NRI due to the lack of established meaningful risk categories in secondary risk prediction. ${ }^{21}$

The IDI corresponds to the absolute change in predicted risk between the old and the new model. It is calculated by subtracting the difference in predicted risk between deceased 
and alive patients in the old model from the difference in predicted risk between deceased and alive patients in the new model. ${ }^{22}$ Additionally, we created optimism-adjusted calibration plots using bootstrapping (repeating the analysis using random resampling with replacement, $n=40$ ), to assess the model fit.

All statistical analyses were performed using Rstudio and the $\mathrm{R}$ software package (version 3.1.2, Vienna, Austria). ${ }^{23} \mathrm{~A}$ two-sided $\mathrm{p}<0.05$ was considered significant.

\section{Results}

\section{Patient characteristics}

During a median follow-up duration of 1.8 years, 77 deaths occurred; 29 of which from cardiovascular events. Patient characteristics are shown in table 1, stratified by mortality status during follow-up. Patients who died during the follow-up period were older (73 vs 63 years, $\mathrm{p}<0.001$ ) and more frequently diabetic than survivors (38vs $22 \%, \mathrm{p}=0.002$ ). Patients who died during follow-up more often had a history of coronary artery bypass grafting surgery, peripheral arterial disease, kidney failure and impaired left ventricular function. Angiotensinconverting enzyme inhibitor (48 vs $34 \%, \mathrm{p}=0.018$ ) and diuretic use was significantly more common in deceased patients ( 55.8 vs $27.4 \%, p<0.001$ ). Median hsTnl levels (22.3 vs 7.1 $\mathrm{ng} / \mathrm{mL}, \mathrm{p}<0.001)$ and NTpro-BNP levels (260.5 vs $83.0 \mathrm{pmol} / \mathrm{L}, \mathrm{p}<0.001)$ were significantly higher in deceased patients. The multivariable adjusted HR of hsTnl and NTpro-BNP for mortality during follow-up were 1.00 [0.93-1.08] per $1000 \mathrm{ng} / \mathrm{mL}$ increase, $\mathrm{p}=0.945$ and 1.27 [1.14-1.42], $\mathrm{p}<0.001$ per $1000 \mathrm{pmol} / \mathrm{L}$ increase, respectively.

\section{Hematological parameters}

Backward Cox regression was performed as described above, providing a panel of 7 hematological parameters. The levels of the hematological parameters for deceased and alive patients and multivariable adjusted hazard ratios (HRs) are displayed in table 2. When adjusted for clinical characteristics and the other selected hematological parameters the hazard ratios (HRs) were as follows: leukocyte count: HR 1.25 [1.12-1.39], $\mathrm{p}<0.001$; reticulocyte mean corpuscular hemoglobin concentration (MCHCr): HR 0.65 [0.50-0.86], $p=0.003 ; R B C$ green (FL1) fluorescence: HR 1.51 [1.15-1.97], $p=0.003 ; \%$ neutrophils: HR 1.37 [1.07-1.75], $p=0.012 ; \%$ large (>120fL) RBCs: HR 1.17 [1.03-1.34], $p=0.019 ; \%$ 
monocytes: HR 1.28 [1.04-1.59], $\mathrm{p}=0.023$ and coefficient of variation (CV) of neutrophil complexity: HR 1.31 [1.03-1.67], $p=0.026$.

\section{All-cause mortality prediction}

We first evaluated the additive predictive value of hsTnl, NTpro-BNP and hematological parameters to a clinical model (table 3 top) for the prediction of all-cause mortality. hsTnl did not improve prediction of mortality in addition to the clinical model (AUCincrease, IDI and cNRI all non-significant). NTpro-BNP on top of the clinical model significantly improved prediction (AUC 0.834 vs $0.818, p=0.019$ ) (Figure 1A) and discrimination (IDI 0.02 [0.00-0.06], $\mathrm{p}=0.040$ ), but not reclassification (cNRI 0.03 [-0.14-0.22], $\mathrm{p}=0.625$ ). The combination of $\mathrm{hsTnl}$ and NTpro-BNP also improved the AUC from 0.818 to 0.834, $p=0.016$ compared with the clinical model alone, but the IDI and cNRI were both nonsignificant.

The addition of hematological parameters to the baseline clinical model (AUC 0.818) significantly improved discrimination (IDI 0.07 [0.03-0.14], p<0.001) and reclassification (cNRI 0.37 [0.19-0.49], $p<0.001)$. The AUC increased to 0.856, $p<0.001$.

We then assessed whether hsTnl, NTpro-BNP or their combination could improve prediction in addition to the clinical model enriched by the hematological parameters (table 3 bottom, Figure 1B). While the AUC increased slightly, albeit non-significantly upon addition of NTpro-BNP (AUC 0.863 vs $0.856, p=0.061$ ), only the combination with hsTnl significantly improved prediction (AUC 0.865, $\mathrm{p}=0.049$ ). Neither hsTnl, NTpro-BNP nor their combination could significantly improve discrimination or reclassification (IDIs and cNRIs all nonsignificant, table 3).

Bootstrapped calibration plots were created (supplemental figure 1) in order to assess goodness-of-fit. Addition of hematological parameters to the clinical model significantly increased the $R^{2}$ (from 0.119 to $0.177, p<0.001$ ), reflecting a better fit. When hsTnl was added to the model, it did not further improve the model fit. However, addition of NTpro-BNP slightly increased model fit $\left(R^{2}\right.$ increased to $\left.0.190, p=0.003\right)$. Optimism-adjusted AUCs provided similar results compared with the AUCs derived from our original models (supplementary figure 3).

Internal Validation 
Models based on a single dataset tend to result in overfitting and overoptimistic estimates. Therefore, our model was internally validated by means of post-estimation shrinkage. The predicted all-cause mortality risk based on the initial model including hematological parameters was grouped into quartiles (Q1 to Q4). Adjusted survival curves are shown in Figure 2. HR for Q3 vs Q1 and Q4 vs Q1 were 6.6 [2.0-21.9], $\mathrm{p}=0.002$ and 9.6 [3.0-31.2], $p<0.001$, respectively. After shrinkage, the HR for Q2 vs Q1 remained nonsignificant. HR for Q3 vs Q1 and Q4 vs Q1 were 3.2 [2.0-5.2], $\mathrm{p}=0.010$ and 4.8 [3.2-7.0], $p<0.001$, respectively. 


\section{Discussion}

Our study shows superiority of hematological parameters over the current clinical standard hsTnl and NTpro-BNP for mortality prediction in CAD patients undergoing coronary angiography. The resulting panel of parameters comprises leukocyte and RBC characteristics. Both, leukocyte and RBC characteristics have previously been reported to serve as strong predictors of mortality in various patient groups.

In cardiovascular disease patients, leukocyte characteristics have been tested and compared with the established marker high-sensitivity C-reactive protein (hsCRP). In stable CAD patients undergoing coronary angiography, neutrophil count was superior to hsCRP in predicting cardiovascular mortality. ${ }^{9}$ In our study, we also included RBC and platelet characteristics as potential predictors of mortality. High platelet reactivity is an independent predictor of future adverse events in myocardial infarction patients and thus seemed to be a logical candidate for testing. ${ }^{24}$ Unexpectedly, none of the platelet characteristics (plateletcrit, mean platelet volume, platelet distribution width, $\mathrm{CV}$ of platelet granularity and reticulated platelet count) in UPOD added any predictive value to the clinical model in our study; mean platelet volume did however predict mortality in univariable analysis in agreement with the literature. ${ }^{25}$ The reasons can be manifold. Our study population mainly consists of stable CAD patients, which have a lower risk of acute fatal thrombotic events due to high platelet reactivity than myocardial infarction patients. Hence, the role of platelets in this cohort might be less relevant. More likely though, the predictive value of platelet characteristics is also reflected by other hematological parameters in our multivariable model, rendering it nonsignificant. Another possibility is that high platelet reactivity is not reflected by their morphology as measured by the hematology analyzers, supporting the need for separate platelet activation testing in high-risk patients. Finally, though our analyses did not investigate such effects, the lack of platelet markers might be due to platelet inhibitors given to the vast majority of patients included in this study.

In contrast, both leukocyte and RBC parameters provided additional predictive value. Monocytes have long been known for their causal role in plaque initiation, progression and destabilization. ${ }^{26}$ As previously shown, their numbers correlate with the presence of 
cardiovascular disease, higher IL-6 levels and predict all-cause and cardiovascular mortality. 27,28

Recent studies have shown the direct involvement of neutrophils in cardiovascular disease. Neutrophil depletion significantly reduced plaque formation in mice. ${ }^{29}$ Furthermore, neutrophils can be found in high numbers in coronary artery autopsy specimens from patients who died of myocardial infarction. ${ }^{30}$ In addition to neutrophil numbers, we found additional predictive value of the variation in neutrophil complexity. This can be regarded as an indication of a 'left shift' or neutrophil activation as frequently seen in acute infections. ${ }^{31}$ Morphological changes of neutrophils have also recently been described to correlate with cardiac function after acute myocardial infarction in a porcine model..$^{32}$ The observed 'left shift' could thus reflect subclinical chronic inflammation due to prevailing coronary atherosclerosis or subacute myocardial ischemia. This is supported by the interaction between neutrophil numbers and morphology on one side and atherosclerosis and ischemia (or vice versa ${ }^{29}$ ) on the other. The exact mechanisms relating these neutrophil characteristics to increased mortality risk however remain to be elucidated.

The role of RBCs in atherosclerosis is less clear. Nevertheless, they harbor significant predictive value in various diseases. In particular RBC distribution width ${ }^{33-35}$ (RDW), which reflects the variation in RBC volume, has been proposed as a powerful risk indicator of mortality.${ }^{11} \mathrm{RBC}$ volume is inversely related with RBC age; young cells are largest, senescent cells are smaller. ${ }^{36}$ RBC characteristics, particularly the \% of large (i.e. young) RBCs that independently predicted mortality in our study concurred with a higher proportion of young erythrocytes (closely related to RDW, $r=0.21, p<0.001$ ). RBC green ( $F L 1)$ fluorescence, which was associated with worse survival in our study reflects the amount of residual RNA in young erythrocytes and reticulocytes. Thus, it could reflect a higher percentage of young $\mathrm{RBC}$, but its clinical relevance remains unclear.

Together with the $\mathrm{MCHCr}$, markers of an immature $\mathrm{RBC}$ population with a low reticulocyte hemoglobin concentration are thus related to a higher risk of mortality in coronary angiography patients.

To our surprise, we did not find any predictive value of hsTnl in our cohort. Highsensitivity troponin $\mathrm{T}$ (hsTnT) has been reported by several other groups as a potential 
predictor of mortality, for example in stroke, ${ }^{37}$ after cardiac surgery ${ }^{38}$ and after elective coronary angiography. ${ }^{5}$ Possibly, this finding is due to differential prognostic properties of hsTnl and hsTnT, as reported by de Antonio et al. ${ }^{39}$

High BNP levels are predictive of adverse events in a population of stable CAD patients $^{6}$ and NTpro-BNP has been shown to predict mortality in an unstable CAD population. ${ }^{40}$ Our study is in line with others, showing that the predictive value of NTpro-BNP for mortality is superior to hsTnl. ${ }^{41}$

Nevertheless, hematological parameters in our study outperformed both NTpro-BNP and hsTnl for prediction of mortality. Apparently, blood cell characteristics provide more prognostic information than the cardiac-specific biomarkers hsTnl and/or NTpro-BNP.

The hematological parameters included in our panel are of leukocyte (total leukocyte count, neutrophil \%, monocyte \% and neutrophil complexity CV) and RBC origin ( $\mathrm{MCHCr}$, RBC green (FL1) fluorescence and \% large RBCs). Leukocyte ${ }^{42}$ and neutrophil counts ${ }^{43}$ have previously been described as prognostic markers for mortality in a population-based cohort. Neutrophil count was not included in our analysis due to its collinearity $(r=0.68, p<0.001)$ with neutrophil \%, which was included as it had a stronger association with mortality.

The reason that blood cells convey more accurate prognostic information than cardiac specific biomarkers could be related to the organ-specificity of hsTnl and NTpro-BNP. The end-point in our study was all-cause mortality, meaning that not all deaths were due to cardiovascular disease or its consequences per se. Hematological parameters could provide a general whole-body overview of an individual's health status and subsequent prognosis. Therefore, we performed an additional analysis for cardiovascular death only. Again, hematological parameters outperformed hsTnl and NTpro-BNP (supplemental figure 2). However, the number of cardiovascular deaths was low $(n=29)$ and these data should thus be interpreted with caution.

In this manuscript the clinical parameters were analyzed as a linear predictor, meaning that the predictive value of these parameters was kept fixed when adding new hematological parameters. This might be a limitation to this study.

Several groups have investigated the relation between statin use and hematological parameters. ${ }^{44,45}$ In our study our results did not change when adding statin use to the 
prediction model, also there was no interaction between statin use and the predictive value of hematological parameters (Pinteraction 0.9).

The past few years many hematological parameters and ratios have been proposed for prediction in cardiovascular diseases, such as the leukocyte count, ${ }^{46}$ monocyte-tolymphocyte ratio, ${ }^{10}$ neutrophil-to-lymphocyte ratio ${ }^{47}$ and so on. While it is unclear which hematological parameter will prove to be the best predictor, it logically makes sense that a panel of several hematological parameters is more accurate than a single parameter or ratio between two, as proposed in this manuscript. External validation of our findings remains indicated.

\section{Conclusions}

Hematological parameters outperform the established biomarkers hsTnl or NTproBNP alone or in combination in predicting all-cause mortality after coronary angiography. Hence, readily available hematological parameters may provide a useful tool to improve current risk prediction algorithms (possibly integrated within the hematology analyzer linked to the electronic patient records) for all-cause mortality in coronary angiography patients. While we put every effort in validating our model internally, external validation is warranted to investigate the clinical use of these parameters and their extension to other cardiovascular disease patient groups.

\section{Disclosures}

None.

\section{Data access}

Access to the data used to perform this research can be requested form the corresponding author.

\section{References}

1. Keller T, Zeller T, Peetz D, et al. Sensitive troponin I assay in early diagnosis of acute myocardial infarction. N Engl J Med. 2009;361:868-77.

2. Hobbs FDR, Davis RC, Roalfe AK, Hare R, Davies MK, Kenkre JE. Reliability of Nterminal pro-brain natriuretic peptide assay in diagnosis of heart failure: cohort study in representative and high risk community populations. BMJ. 2002;324:1498. 
3. Bonaca MP, O'Malley RG, Murphy SA, et al. Prognostic performance of a highsensitivity assay for cardiac troponin I after non-ST elevation acute coronary syndrome: Analysis from MERLIN-TIMI 36. Eur Heart J Acute Cardiovasc Care. 2014; 4: $431-40$.

4. Grodin JL, Neale S, Wu Y, Hazen SL, Tang WHW. Prognostic Comparison of Different Sensitivity Cardiac Troponin Assays in Stable Heart Failure. Am J Med. Elsevier Inc; 2015;128:276-82.

5. Hochholzer W, Valina CM, Stratz C, et al. High-sensitivity cardiac troponin for risk prediction in patients with and without coronary heart disease. Int $\mathrm{J}$ Cardiol. 2014;176:444-9.

6. Schnabel R, Lubos E, Rupprecht HJ, et al. B-type natriuretic peptide and the risk of cardiovascular events and death in patients with stable angina: Results from the AtheroGene study. J Am Coll Cardiol. 2006;47:552-8.

7. James SK, Lindahl B, Siegbahn A, et al. N-terminal pro-brain natriuretic peptide and other risk markers for the separate prediction of mortality and subsequent myocardial infarction in patients with unstable coronary artery disease: A GUSTO-IV Substudy. Circulation. 2003;108:275-81.

8. Yildirir A, Acikel S, Ertan C, Aydinalp A, Ozin B, Muderrisoglu H. Value of periprocedural B-type natriuretic peptide levels in predicting cardiac events after elective percutaneous coronary intervention. Acta Cardiol. 2008;63:47-52.

9. ó Hartaigh B, Bosch JA, Thomas GN, et al. Which leukocyte subsets predict cardiovascular mortality? From the LUdwigshafen RIsk and Cardiovascular Health (LURIC) Study. Atherosclerosis. 2012;224:161-9.

10. Gijsberts CM, Ellenbroek GH, Ten Berg MJ, et al. Routinely analyzed leukocyte characteristics improve prediction of mortality after coronary angiography. Eur J Prev Cardiol. 2015 Dec 7;pii: 2047487315621832.

11. Gijsberts CM, den Ruijter HM, de Kleijn DPV, et al. Hematological Parameters Improve Prediction of Mortality and Secondary Adverse Events in Coronary Angiography Patients. Medicine (Baltimore). 2015;94:e1992.

12. Müller R, Mellors I, Johannessen B, et al. European multi-center evaluation of the Abbott Cell-Dyn sapphire hematology analyzer. Lab Hematol. 2006;12:15-31.

13. ten Berg MJ, Huisman A, van den Bemt PMLA, Schobben AFAM, Egberts ACG, van Solinge WW. Linking laboratory and medication data: new opportunities for pharmacoepidemiological research. Clin Chem Lab Med. 2007;45:13-9.

14. Gijsberts CM, Seneviratna A, de Carvalho LP, et al. Ethnicity Modifies Associations between Cardiovascular Risk Factors and Disease Severity in Parallel Dutch and Singapore Coronary Cohorts. Devaney J, editor. PLoS One. 2015 6;10:e0132278.

15. Harris PJ, Behar VS, Conley MJ, et al. The prognostic significance of $50 \%$ coronary stenosis in medically treated patients with coronary artery disease. Circulation. 1980;62:240-8.

16. Lam SW, Leenen LPH, Van Solinge WW, Hietbrink F, Huisman A. Evaluation of hematological parameters on admission for the prediction of 7-day in-hospital mortality in a large trauma cohort. Clin Chem Lab Med. 2011;49:493-9.

17. Pavlou M, Ambler G, Seaman SR, et al. How to develop a more accurate risk prediction model when there are few events. BMJ. 2015;351:h3868.

18. Dunkler D, Heinze G. Package "shrink " [Internet]. CRAN. 2014 [cited 2015 Oct 14]. p. 1-15. Available from: https://cran.r-project.org/web/packages/shrink/shrink.pdf

19. Uno H, Tian L, Cai T, Kohane IS, Wei LJ. A unified inference procedure for a class of measures to assess improvement in risk prediction systems with survival data. Stat Med. 2013;32:2430-42. 
20. Pencina MJ, D'Agostino RB, Steyerberg EW. Extensions of net reclassification improvement calculations to measure usefulness of new biomarkers. Stat Med. 2011;30:11-21.

21. Sniderman AD, D'Agostino RB, Pencina MJ. The Role of Physicians in the Era of Predictive Analytics. JAMA. 2015;314:25-6.

22. Steyerberg EW, Vickers AJ, Cook NR, et al. Assessing the Performance of Prediction Models. Epidemiology. 2010;21:128-38.

23. R Core Team. R: A Language and Environment for Statistical Computing. Vienna, Austria; 2014. R Foundation for Statistical Computing. URL: http://www.R-project.org/.

24. Campo G, Valgimigli M, Gemmati D, et al. Value of platelet reactivity in predicting response to treatment and clinical outcome in patients undergoing primary coronary intervention: insights into the STRATEGY Study. J Am Coll Cardiol. 2006 ;48):217885.

25. Renga B, Scavizzi F. Platelets and cardiovascular risk. Acta Cardiol. 2017;72:2-8.

26. Ghattas A, Griffiths HR, Devitt A, Lip GYH, Shantsila E. Monocytes in coronary artery disease and atherosclerosis: where are we now? J Am Coll Cardiol. 2013;62:1541-51.

27. Madjid M, Fatemi O. Components of the complete blood count as risk predictors for coronary heart disease: in-depth review and update. Tex Heart Inst J. 2013;40):17-29.

28. Kato A, Takita T, Furuhashi M, Maruyama Y, Kumagai H, Hishida A. Blood monocyte count is a predictor of total and cardiovascular mortality in hemodialysis patients. Nephron Clin Pract. 2008;110:c235-43.

29. Zernecke A, Bot I, Djalali-Talab Y, et al. Protective role of CXC receptor $4 / C X C$ ligand 12 unveils the importance of neutrophils in atherosclerosis. Circ Res. 2008;102:20917.

30. Naruko T, Ueda M, Haze K, et al. Neutrophil infiltration of culprit lesions in acute coronary syndromes. Circulation. 2002;106:2894-900.

31. Seebach JD, Morant R, Rüegg R, Seifert B, Fehr J. The diagnostic value of the neutrophil left shift in predicting inflammatory and infectious disease. Am J Clin Pathol. 1997; 107:582-91.

32. van Hout GPJ, van Solinge WW, Gijsberts CM, et al. Elevated mean neutrophil volume represents altered neutrophil composition and reflects damage after myocardial infarction. Basic Res Cardiol. 2015;110:58.

33. Tsuboi S, Miyauchi K, Kasai T, et al. Impact of red blood cell distribution width on longterm mortality in diabetic patients after percutaneous coronary intervention. Circ J. 2013;77:456-61.

34. Núñez J, Núñez E, Rizopoulos D, et al. Red blood cell distribution width is longitudinally associated with mortality and anemia in heart failure patients. Circ J. 2014;78:410-8.

35. Perlstein TS, Weuve J, Pfeffer M a, Beckman J a. Red blood cell distribution width and mortality risk in a community-based prospective cohort. Arch Intern Med. 2009;169:588-94.

36. Patel HH, Patel HR, Higgins JM. Modulation of red blood cell population dynamics is a fundamental homeostatic response to disease. Am J Hematol. 2015;90:422-8.

37. Maoz A, Rosenberg S, Leker RR. Increased High-Sensitivity Troponin-T Levels Are Associated with Mortality After Ischemic Stroke. J Mol Neurosci. 2015; 57:160-5.

38. Lurati Buse GAL, Bolliger D, Seeberger E, et al. Troponin T and B-type natriuretic peptide after on-pump cardiac surgery: prognostic impact on 12-month mortality and major cardiac events after adjustment for postoperative complications. Circulation. 
2014;130:948-57.

39. de Antonio M, Lupón J, Galán A, et al. Head-to-head comparison of high-sensitivity troponin $\mathrm{T}$ and sensitive-contemporary troponin I regarding heart failure risk stratification. Clin Chim Acta. 2013;426:18-24.

40. Omland $\mathrm{T}$, Persson $\mathrm{A}, \mathrm{Ng} \mathrm{L}$, et al. $\mathrm{N}$-terminal pro-B-type natriuretic peptide and longterm mortality in acute coronary syndromes. Circulation. 2002;106:2913-8.

41. Nigro N, Wildi K, Mueller C, et al. BNP but Not s-cTnln Is Associated with Cardioembolic Aetiology and Predicts Short and Long Term Prognosis after Cerebrovascular Events. PLoS One. 2014;9:e102704.

42. Karino S, Willcox BJ, Fong K, Lo S, Abbott R, Masaki KH. Total and differential white blood cell counts predict eight-year incident coronary heart disease in elderly Japanese-American men: The Honolulu Heart Program. Atherosclerosis. 2014 ;238:153-8.

43. Guasti L, Dentali F, Castiglioni L, et al. Neutrophils and clinical outcomes in patients with acute coronary syndromes and/or cardiac revascularization: A systematic review on more than 34,000 subjects. Thromb Haemost. 2011;106:591-9.

44. Jaumdally RJ, Lip GYH, Varma C, Blann AD. Impact of high-dose atorvastatin on endothelial, platelet, and angiogenic indices: effect of ethnicity, cardiovascular disease, and diabetes. Angiology. 2011;62:571-8.

45. Akın F, Ayça B, Köse N, et al. Effect of Atorvastatin on Hematologic Parameters in Patients With Hypercholesterolemia. Angiology. 2013;64:621-5.

46. Madjid M, Awan I, Willerson JT, Casscells SW. Leukocyte count and coronary heart disease: implications for risk assessment. J Am Coll Cardiol. 2004;44:1945-56.

47. Wang X, Zhang G, Jiang X, Zhu H, Lu Z, Xu L. Neutrophil to lymphocyte ratio in relation to risk of all-cause mortality and cardiovascular events among patients undergoing angiography or cardiac revascularization: a meta-analysis of observational studies. Atherosclerosis. 2014;234:206-13. 
Figure 1. ROC plots of hematological parameters, hsTnl and NTpro-BNP; A) in addition to a clinical model for the prediction of all-cause mortality, B) in addition to a clinical model plus hematological parameters for the prediction of all-cause mortality

A) ROC plots of the clinical model and of the clinical model extended with hsTnl, NTpro-BNP (or both) and hematological parameters for the prediction of all-cause mortality during 2 years of follow-up. B) ROC plots of the clinical model plus hematological parameters and of that model extended with hsTnl, NTpro-BNP or both for the prediction of all-cause mortality during 2 years of follow-up. "Clin + H" stands for clinical model plus hematological parameters.

Abbreviations: $\mathrm{ROC}=$ receiver operating characteristics, $\mathrm{hsTnl}=$ high-sensitivity troponin $\mathrm{I}$, NTpro-BNP $=\mathrm{N}$-terminal pro-brain natriuretic peptide. 
Figure 2. Survival plot showing survival by quartiles of predicted risk based on hematological parameters, adjusted for clinical characteristics

The predicted risk of all-cause mortality based on hematological parameters, was grouped into 4 quartiles (Q1 to Q4). See methods section for more detailed explanation. The multivariable adjusted survival in these quartiles was plotted using Cox regression analysis. The HR for Q2 vs Q1 was not significant, for Q3 vs Q1 it was 6.5 [2.0-21.8], $\mathrm{p}=0.002$; the HR for $\mathrm{Q} 4$ vs $\mathrm{Q} 1$ was 11.8 [3.6-38.1], $\mathrm{p}<0.001$. Abbreviations: $\mathrm{HR}=$ hazard ratio. 


\section{Supplemental figure 1. Calibration plots of prediction models}

The predicted $v s$ the observed risk is shown for the clinical model, the clinical model plus hematology, the clinical model plus hematology and hsTnl and the clinical model plus hematology and hsTnl and NTpro-BNP. The model fit is significantly improved by the addition of hematological parameters. Addition of hsTnl results in no improvement. Addition of NTproBNP however does slightly improve model fit further. *p-value from Wald-test comparing the Cox model to the previous one (without the added marker in question).

Abbreviations: hsTnl $=$ high-sensitivity troponin $\mathrm{I}, \mathrm{NTpro-BNP}=\mathrm{N}$-terminal pro-brain natriuretic peptide. 
Supplemental figure 2. ROC plots of hematological parameters, hsTnl and NTpro-BNP in addition to a clinical model for the prediction of cardiovascular mortality ROC plots of the clinical model and of the clinical model extended with hsTnl, NTpro-BNP (or both) and hematological parameters for the prediction of cardiovascular mortality during 2 years of follow-up. Abbreviations: $\mathrm{ROC}=$ receiver operating characteristics, $\mathrm{hs} T \mathrm{nl}=$ highsensitivity troponin I, NTpro-BNP = N-terminal pro-brain natriuretic peptide. 
Supplemental figure 3. Optimism-adjusted AUCs for the models used in this manuscript.

These adjusted AUCs were calculated using a bootstrapping approach $(n=100)$. Original and adjusted AUCs are shown in the red (adjusted AUC) and blue lines (original AUC). Abbreviations: $A \cup C=$ area under the curve (from receiver operating characteristics analysis). 
Table 1. Baseline characteristics of UCORBIO patients.

\begin{tabular}{|c|c|c|c|c|}
\hline & Overall & Alive & Deceased & $p$ \\
\hline $\mathrm{n}$ & 1913 & 1836 & 77 & \\
\hline Age, mean (sd) & $63.7(10.9)$ & $63.3(10.8)$ & $72.5(8.7)$ & $<0.001$ \\
\hline $\operatorname{Sex}(\%)$ & 73.9 & 73.9 & 72.7 & 0.921 \\
\hline BMI, mean (sd) & $27.2(4.5)$ & $27.2(4.5)$ & $26.6(4.8)$ & 0.281 \\
\hline Diabetes (\%) & 22.7 & 22.1 & 37.7 & 0.002 \\
\hline Hypertension (\%) & 59.0 & 58.8 & 63.6 & 0.464 \\
\hline Hypercholesterolemia (\%) & 48.0 & 48.4 & 39.0 & 0.133 \\
\hline Smoking (\%) & & & & 0.068 \\
\hline Active smoker & 25.6 & 25.9 & 19.5 & \\
\hline Ex-smoker & 26.5 & 26.0 & 37.7 & \\
\hline Non smoker & 47.9 & 48.1 & 42.9 & \\
\hline History of ACS (\%) & 30.6 & 30.3 & 37.7 & 0.215 \\
\hline History of $\mathrm{PCl}(\%)$ & 27.7 & 27.8 & 23.4 & 0.468 \\
\hline History of CABG (\%) & 10.5 & 10.1 & 18.2 & 0.038 \\
\hline History of CVA (\%) & 10.5 & 10.2 & 16.9 & 0.091 \\
\hline History of PAD (\%) & 11.7 & 11.3 & 20.8 & 0.018 \\
\hline Kidney failure (\%) & 2.8 & 2.5 & 10.4 & $<0.001$ \\
\hline EF (\%) & & & & $<0.001$ \\
\hline Normal & 57.0 & 58.2 & 32.4 & \\
\hline Mildly impaired & 23.2 & 23.1 & 25.0 & \\
\hline Impaired & 12.1 & 11.7 & 20.6 & \\
\hline Poor & 7.6 & 6.9 & 22.1 & \\
\hline Aspirin (\%) & 58.1 & 58.0 & 59.7 & 0.859 \\
\hline Clopidogrel (\%) & 20.8 & 20.9 & 18.2 & 0.658 \\
\hline Beta-blocker (\%) & 54.5 & 54.3 & 59.7 & 0.409 \\
\hline ACE inhibitor (\%) & 34.8 & 34.3 & 48.1 & 0.018 \\
\hline Statin (\%) & 61.6 & 61.5 & 63.6 & 0.802 \\
\hline Diuretic (\%) & 28.6 & 27.4 & 55.8 & $<0.001$ \\
\hline \multicolumn{5}{|l|}{ Coronary Angiography } \\
\hline Indication (\%) & & & & 0.222 \\
\hline Stable CAD & 56.5 & 56.5 & 55.8 & \\
\hline UAP & 9.7 & 9.8 & 6.5 & \\
\hline Infarction & 28.4 & 28.4 & 27.3 & \\
\hline Other & 5.5 & 5.3 & 10.4 & \\
\hline Severity of CAD (\%) & & & & 0.566 \\
\hline No CAD & 6.3 & 6.3 & 6.5 & \\
\hline Minor CAD & 15.2 & 15.0 & 19.5 & \\
\hline Single vessel disease & 33.7 & 33.9 & 27.3 & \\
\hline Multi vessel disease & 44.9 & 44.8 & 46.8 & \\
\hline Procedure (\%) & & & & 0.613 \\
\hline Conservative & 31.3 & 31.1 & 36.4 & \\
\hline $\mathrm{PCl}$ & 62.6 & 62.8 & 58.4 & \\
\hline CABG & 6.1 & 6.1 & 5.2 & \\
\hline hsTnl (ng/mL, median [IQR]) & $7.4[3.7,30.1]$ & $7.1[3.6,27.4]$ & $22.3[5.1,65.9]$ & $<0.001$ \\
\hline NT proBNP (pmol/L, median [IQR]) & $86.4[33.4,210.5]$ & $83.0[32.6,199.8]$ & $260.5[89.5,598.4]$ & $<0.001$ \\
\hline FU in years (median [IQR]) & $1.8[1.0,2.6]$ & $1.7[1.0,2.5]$ & $2.2[1.8,2.9]$ & $<0.001$ \\
\hline
\end{tabular}

Abbreviations: UCORBIO = Utrecht Coronary Biobank, $\mathrm{BMI}=$ body mass index, $\mathrm{ACS}=$ acute

coronary syndrome, $\mathrm{PCI}=$ percutaneous coronary intervention, $\mathrm{CABG}=$ coronary artery bypass grafting, $\mathrm{CVA}=$ cerebrovascular accident, $\mathrm{PAD}=$ peripheral arterial disease, $\mathrm{EF}=$ ejection fraction, $\mathrm{ACE}=$ angiotensin-converting enzyme, UAP $=$ unstable angina pectoris, $\mathrm{FU}$ $=$ follow-up, $\mathrm{CAD}=$ coronary artery disease, $\mathrm{hsTnl}=$ high-sensitivity troponin I, NTpro-BNP $\mathrm{N}$-terminal pro-brain natriuretic peptide. 
Table 2. Characteristics of hematological parameters included in the prognostic set.

\begin{tabular}{|c|c|c|c|c|c|}
\hline & Value Alive & Value Deceased & $\begin{array}{r}p \text {-value } \\
\text { difference }\end{array}$ & HR $(95 \% \mathrm{Cl})$ & p-value \\
\hline Leukocyte count (10^9 cells/L) & $2.32[1.91,2.86]$ & $2.42[1.94,3.11]$ & 0.224 & $1.25(1.12-1.39)$ & $<0.001$ \\
\hline $\mathrm{MCHCr}(\mathrm{mmol} / \mathrm{L})$ & $15.73[15.28,16.22]$ & $15.24[14.83,15.68]$ & $<0.001$ & $0.65(0.50-0.86)$ & 0.003 \\
\hline RBC green (FL1) fluorescence (AU) & $17.52[17.01,18.03]$ & $17.76[17.30,18.12]$ & 0.011 & $1.51(1.15-1.97)$ & 0.003 \\
\hline$\%$ neutrophils (\%) & $5.97[5.32,6.60]$ & $6.29[5.74,7.00]$ & 0.001 & $1.37(1.07-1.75)$ & 0.012 \\
\hline$\%$ large* RBCs (\%) & $0.71[0.46,1.08]$ & $1.03[0.71,2.00]$ & $<0.001$ & $1.17(1.03-1.34)$ & 0.019 \\
\hline$\%$ monocytes (\%) & $3.18[2.65,3.81]$ & $3.58[2.58,4.19]$ & 0.075 & $1.28(1.04-1.59)$ & 0.023 \\
\hline Neutrophil complexity CV (\%) & $7.27[6.70,7.89]$ & $7.31[6.65,8.31]$ & 0.377 & $1.31(1.03-1.67)$ & 0.026 \\
\hline
\end{tabular}

Medians and interquartile ranges of hematological parameters are shown for alive and deceased patients. The multivariable adjusted hazard ratios for all-cause mortality are shown for each 1-SD increase the hematological parameter and derived from a model containing: age, sex, diabetes, hypercholesterolemia, smoking status, indication for angiography, angiographic $\mathrm{CAD}$ severity, history of $\mathrm{PCl}$, history of $\mathrm{ACS}$, kidney failure, treatment following angiography and the other hematological parameters shown in the table. The p-values in the far right column correspond to significance of the multivariable adjusted hazard ratios in the prior column.

Abbreviations: $\mathrm{MCHCr}=$ reticulocyte mean corpuscular hemoglobin concentration, $\mathrm{RBC}=$ red blood cell, $\mathrm{AU}=$ arbitrary units, $\mathrm{CV}=$ coefficient of variation. ${ }^{*}>120 \mathrm{fL}$. 
Table 3. Measures of improvement of all-cause mortality prediction.

\begin{tabular}{lcrrr} 
In addition to clinical characteristics & $\begin{array}{r}\text { IDI } \\
\text { p-value } \\
\text { IDI }\end{array}$ & $\begin{array}{r}\text { cNRI } \\
\text { p-value } \\
\text { cNRI }\end{array}$ \\
\hline Hematology & $0.07(0.03-0.14)$ & $<0.001$ & $0.37(0.19-0.49)$ & $<0.001$ \\
hsTnl & $0.00(0.00-0.00)$ & 0.817 & $-0.07(-0.16-0.20)$ & 0.970 \\
NTpro-BNP & $0.02(0.00-0.06)$ & 0.040 & $0.03(-0.14-0.22)$ & 0.625 \\
hsTnl + NTpro-BNP & $0.02(0.00-0.07)$ & 0.066 & $0.02(-0.13-0.20)$ & 0.671 \\
Hematology + hsTnl + NTpro-BNP & $0.09(0.05-0.17)$ & $<0.001$ & $0.44(0.24-0.53)$ & 0.007 \\
In addition to clinical and hematological parameters & & & \\
\hline hsTnl & $0.01(0.00-0.02)$ & 0.113 & $0.16(-0.18-0.31)$ & 0.292 \\
NTpro-BNP & $0.01(-0.01-0.06)$ & 0.186 & $-0.08(-0.25-0.14)$ & 0.777 \\
hsTnl + NTpro-BNP & $0.02(0.00-0.06)$ & 0.093 & $-0.03(-0.23-0.27)$ & 1 \\
\hline
\end{tabular}

Abbreviations: $\mid \mathrm{IDI}=$ integrated discrimination improvement, $\mathrm{cNRI}=$ continuous net reclassification improvement, hsTnl $=$ high-sensitivity troponin I, NTpro-BNP $-\mathrm{N}$-terminal probrain natriuretic peptide. 


\section{Supplemental table 1. Parameters available and selected in the Utrecht Patient}

\section{Oriented Database (UPOD).}

\begin{tabular}{lr} 
UPOD parameters & $\begin{array}{r}\text { Not intercorrelated } \\
\text { (selected for further analysis) }\end{array}$ \\
\hline Leukocyte count & $\mathrm{X}$ \\
Neutrophil count & $\mathrm{X}$ \\
Lymphocyte count & \\
Monocyte count & $\mathrm{X}$ \\
Eosinophil count & $\mathrm{X}$ \\
Basophil count & $\mathrm{X}$ \\
Neutrophil \% & $\mathrm{X}$ \\
Lymphocyte \% & $\mathrm{X}$ \\
Monocyte \% & \\
Eosinophil \% & $\mathrm{X}$ \\
Basophil \% & $\mathrm{X}$ \\
Red blood cell count & $\mathrm{X}$ \\
Hemoglobin concentration & \\
Mean corpuscular volume & \\
Red blood cell distribution width & \\
Mean corpuscular hemoglobin & $\mathrm{X}$ \\
Mean corpuscular hemoglobin concentration & $\mathrm{X}$ \\
Hematocrit & $\mathrm{X}$ \\
Platelet count & $\mathrm{X}$ \\
Mean platelet volume &
\end{tabular}

Reticulocyte\%

Immature reticulocyte fraction

Mean neutrophil cell size

Mean neutrophil complexity

Mean neutrophil lobularity

Mean neutrophil granularity

Mean neutrophil red fluorescence

CV of neutrophil cell size

$\mathrm{CV}$ of neutrophil complexity

$\mathrm{CV}$ of neutrophil lobularity

$\mathrm{CV}$ of neutrophil granularity

CV of neutrophil red fluorescence

Mean lymphocyte cell size

Mean lymphocyte complexity

$\mathrm{CV}$ of lymphocyte cell size

CV of lymphocyte complexity

Mean platelet complexity

Mean platelet granularity

$\mathrm{CV}$ of platelet complexity

CV of platelet granularity

Mean red blood cell complexity

$\mathrm{CV}$ of red blood cell complexity

Mean red blood cell green (FL1) fluorescence

$\mathrm{CV}$ of red blood cell green (FL1) fluorescence

Reticulocyte mean corpuscular hemoglobin concentration

Hemoglobin distribution width

Reticulocyte mean corpuscular hemoglobin

Reticulocyte mean corpuscular volume

$\%$ red blood cells with hemoglobin concentration $<28 \mathrm{~g} / \mathrm{dL}$

$\%$ red blood cells with hemoglobin concentration $>41 \mathrm{~g} / \mathrm{dL}$

$\%$ red blood cells with volume $>120 \mathrm{fL}$

$\%$ red blood cells with volume $<60 \mathrm{fL}$

analysis and therefore selected for further analysis.

$\mathrm{CV}=$ coefficient of variation 